\title{
Convergence theorems for common solutions of various problems with nonlinear mapping
}

Kyung Soo Kim", Jong Kyu Kim² and Won Hee Lim²

\section{*Correspondence:}

kksmj@kyungnam.ac.kr

${ }^{1}$ Graduate School of Education,

Mathematics Education, Kyungnam

University, Changwon, Kyungnam

631-701, Republic of Korea

Full list of author information is

available at the end of the article

\begin{abstract}
In this paper, motivated and inspired by Zegeye and Shahzad (Nonlinear Anal. 70:2707-2716, 2009), Qin et al. (J. Comput. Appl. Math. 225(1):20-30, 2009) and Kimura and Takahashi (J. Math. Anal. Appl. 357:356-363, 2009), we introduce a new hybrid projection iterative scheme that converges strongly to a common element of the solution set of a generalized mixed equilibrium problem, the solution set of a variational inequality problem, and the set of common fixed points for a family of hemi-relatively nonexpansive mappings in a Banach space.
\end{abstract}

MSC: 47H10; 47J20; 49J40

Keywords: common fixed point; generalized mixed equilibrium problem; generalized projection; hemi-relatively nonexpansive; variational inequality problem

\section{Introduction}

A real Banach space $E$ is said to be strictly convex if $\left\|\frac{x+y}{2}\right\|<1$ for all $x, y \in E$ with $\|x\|=\|y\|=$ 1 and $x \neq y$. It is said to be uniformly convex if $\lim _{n \rightarrow \infty}\left\|x_{n}-y_{n}\right\|=0$ for any two sequences $\left\{x_{n}\right\}$ and $\left\{y_{n}\right\}$ in $E$ such that $\left\|x_{n}\right\|=\left\|y_{n}\right\|=1$ and $\lim _{n \rightarrow \infty}\left\|\frac{x_{n}+y_{n}}{2}\right\|=1$. It is known that a uniformly convex Banach space is reflexive and strictly convex. Let $U=\{x \in E:\|x\|=1\}$ be the unit sphere of $E$. Then the Banach space $E$ is said to be smooth if

$$
\lim _{t \rightarrow 0} \frac{\|x+t y\|-\|x\|}{t}
$$

exists for each $x, y \in U$. It is said to be uniformly smooth if the limit is attained uniformly for $x, y \in E$.

Let $E$ be a real Banach space with the norm $\|\cdot\|$, and let $E^{*}$ denote the dual space of $E$. We denote by $J$ the normalized duality mapping from $E$ to $2^{E^{*}}$ defined by

$$
J x=\left\{f^{*} \in E^{*}:\left\langle x, f^{*}\right\rangle=\|x\|^{2}=\left\|f^{*}\right\|^{2}\right\}
$$

where $\langle\cdot, \cdot\rangle$ denotes the generalized duality pairing. It is well known that if $E^{*}$ is strictly convex, then $J$ is single-valued, and if $E$ is uniformly smooth, then $J$ is uniformly norm-tonorm continuous on a bounded subset of $E$. Moreover, if $E$ is a reflexive and strictly convex Banach space with a strictly convex dual, then $J^{-1}$ is single-valued, one-to-one, surjective

\section{黛 Springer}

@2014 Kim et al.; licensee Springer. This is an Open Access article distributed under the terms of the Creative Commons Attribution License (http://creativecommons.org/licenses/by/2.0), which permits unrestricted use, distribution, and reproduction in any medium, provided the original work is properly cited. 
and it is the duality mapping from $E^{*}$ into $E$ and thus $J J^{-1}=I_{E^{*}}$ and $J^{-1} J=I_{E}$ (see [1]). We note that $J$ is the identity mapping in a Hilbert space.

A mapping $A: D(A) \subset E \rightarrow E^{*}$ is said to be monotone if for each $x, y \in D(A)$,

$$
\langle x-y, A x-A y\rangle \geq 0 .
$$

A mapping $A$ is said to be $\gamma$-inverse strongly monotone if there exists a positive real number $\gamma>0$ such that

$$
\langle x-y, A x-A y\rangle \geq \gamma\|A x-A y\|^{2}, \quad \forall x, y \in D(A) .
$$

If a mapping $A$ is $\gamma$-inverse strongly monotone, then it is Lipschitz continuous with constant $\frac{1}{\gamma}$, i.e.,

$$
\|A x-A y\| \leq \frac{1}{\gamma}\|x-y\|, \quad x, y \in D(A) .
$$

A mapping $A$ is said to be strongly monotone, if for each $x, y \in D(A)$, there exists $k \in(0,1)$ such that

$$
\langle x-y, A x-A y\rangle \geq k\|x-y\|^{2} .
$$

A monotone mapping $A$ is said to be maximal if its graph $G(A)=\{(x, y): y \in A x\}$ is not properly contained in the graph of any other monotone mapping. It is known that the monotone mapping $A$ is maximal if and only if for $\left(x, x^{*}\right) \in E \times E^{*}$,

$$
\left\langle x-y, x^{*}-y^{*}\right\rangle \geq 0
$$

for every $\left(y, y^{*}\right) \in G(A)$ implies that $x^{*} \in A x$.

Let $C$ be a nonempty, closed convex subset of a Banach space $E$. For a bifunction $\theta$ : $C \times C \rightarrow \mathbb{R}$, we assume that $\theta$ satisfies the following conditions:

(E1) $\theta(x, x)=0$ for all $x \in C$;

(E2) $\theta$ is monotone, i.e., $\theta(x, y)+\theta(y, x) \leq 0$ for all $x, y \in C$;

(E3) for each $x, y, z \in C$,

$$
\lim _{t \downarrow 0} \theta(t z+(1-t) x, y) \leq \theta(x, y) ;
$$

(E4) for each $x \in C$, the function $y \mapsto \theta(x, y)$ is convex and lower semi-continuous.

The generalized mixed equilibrium problem is to find $x \in C$ such that

$$
\theta(x, y)+\psi(y)-\psi(x)+\langle A x, y-x\rangle \geq 0, \quad \forall y \in C,
$$

where $\psi$ is a lower semicontinuous and convex function. The set of solutions of problem (1.1) is denoted by GMEP. Recently, Zhang [2] considered this problem. Some special cases of problem (1.1) are stated as follows. 
If $A=0$, then problem (1.1) reduces to the following mixed equilibrium problem of finding $x \in C$ such that

$$
\theta(x, y)+\psi(y)-\psi(x) \geq 0, \quad \forall y \in C,
$$

which was considered by Ceng and Yao [3].

If $\psi=0$, then problem (1.1) reduces to the following generalized equilibrium problem of finding $x \in C$ such that

$$
\theta(x, y)+\langle A x, y-x\rangle \geq 0, \quad \forall y \in C,
$$

which was studied in [4].

If $\psi=0$ and $A=0$, then problem (1.1) reduces to the following equilibrium problem of finding $x \in C$ such that

$$
\theta(x, y) \geq 0, \quad \forall y \in C
$$

The set of solutions of problem (1.2) is denoted by $E P$.

If $\theta=0$ and $\psi=0$, then problem (1.1) reduces to the following classical variational inequality problem of finding $x \in C$ such that

$$
\langle A x, y-x\rangle \geq 0, \quad \forall y \in C
$$

The set of solutions of problem (1.3) is denoted by $V I(C, A)$.

Equilibrium problems, which were introduced in [5] in 1994, have had great impact and influence on the development of several branches of pure and applied sciences. They include numerous problems in economics, finance, physics, network, elasticity, optimization, variational inequalities, minimax problems, and semigroups; see, for instance, [2-4, 6-17] and the references therein.

As well known, if $C$ is a nonempty, closed and convex subset of a Hilbert space $H$ and $P_{C}: H \rightarrow C$ is the metric projection of $H$ onto $C$, then $P_{C}$ is nonexpansive. This fact actually characterizes Hilbert spaces and, consequently, it is not true to more general Banach spaces. In this connection, Alber [18] introduced a generalized projection operator $\Pi_{C}$ in a Banach space $E$ which is an analogue of the metric projection in Hilbert spaces. Consider the functional defined by

$$
\phi(x, y)=\|x\|^{2}-2\langle x, J y\rangle+\|y\|^{2} \quad \text { for } x, y \in E .
$$

Observe that, in a Hilbert space $H$, (1.4) reduces to

$$
\phi(x, y)=\|x-y\|^{2} \quad \text { for } x, y \in H .
$$

The generalized projection $\Pi_{C}: E \rightarrow C$ is a mapping that assigns an arbitrary point $x \in E$ to the minimum point of the functional $\phi(x, y)$, that is, $\Pi_{C} x=\bar{x}$, where $\bar{x}$ is the solution to the minimization problem

$$
\phi(\bar{x}, x)=\inf _{y \in C} \phi(y, x)
$$


The existence and uniqueness of the mapping $\Pi_{C}$ follows from the properties of the functional $\phi(x, y)$ and strict monotonicity of the mapping $J$ (see, for example, [18] and [19]). In a Hilbert space, $\Pi_{C}=P_{C}$. It is obvious from the definition of the function $\phi$ that:

(1) $(\|x\|-\|y\|)^{2} \leq \phi(x, y) \leq(\|x\|+\|y\|)^{2}$ for all $x, y \in E$.

(2) $\phi(x, y)=\phi(x, z)+\phi(z, y)+2\langle x-z, J z-J y\rangle$ for all $x, y, z \in E$.

(3) $\phi(x, y)=\langle x, J x-J y\rangle+\langle y-x, J y\rangle \leq\|x\|\|J x-J y\|+\|y-x\|\|y\|$ for all $x, y \in E$.

(4) If $E$ is a reflexive, strictly convex and smooth Banach space, then, for all $x, y \in E$,

$$
\phi(x, y)=0 \quad \text { if and only if } \quad x=y .
$$

Remark 1.1 In (4), it is sufficient to show that if $\phi(x, y)=0$ then $x=y$. In fact, from (1) we have $\|x\|=\|y\|$. This implies that $\langle x, J y\rangle=\|x\|^{2}=\|J y\|^{2}$. From the definition of $J$, we have $J x=J y$. Therefore, we have $x=y$. For more details, see [1].

Let $C$ be a nonempty closed and convex subset of $E$, and let $T$ be a mapping from $C$ into itself. We denote by $F(T)$ be the set of fixed points of $T$. A point $p$ in $C$ is said to be a weak asymptotic fixed point of $T$ [20] if $C$ contains a sequence $\left\{x_{n}\right\}$ which converges weakly to $p$ such that $\lim _{n \rightarrow \infty}\left(T x_{n}-x_{n}\right)=0$. The set of asymptotic fixed points of $T$ will be denoted by $\hat{F}(T)$. A mapping $T$ from $C$ into itself is called relatively nonexpansive [21-23] if $\hat{F}(T)=F(T)$ and $\phi(p, T x) \leq \phi(p, x)$ for all $x \in C$ and $p \in F(T)$. The asymptotic behavior of relatively nonexpansive mappings was studied in [21,22] and [12].

A point $p$ in $C$ is said to be a strong asymptotic fixed point of $T$ if $C$ contains a sequence $\left\{x_{n}\right\}$ which converges strongly to $p$ such that $\lim _{n \rightarrow \infty}\left(T x_{n}-x_{n}\right)=0$. The set of strong asymptotic fixed points of $T$ is denoted by $\tilde{F}(T)$. A mapping $T$ from $C$ into itself is called relatively weak nonexpansive if $\tilde{F}(T)=F(T)$ and $\phi(p, T x) \leq \phi(p, x)$ for all $x \in C$ and $p \in F(T)$. A mapping $T$ is called hemi-relatively nonexpansive if $F(T) \neq \emptyset$ and $\phi(p, T x) \leq \phi(p, x)$ for all $x \in C$ and $p \in F(T)$.

Remark 1.2 (1) It is obvious that a relatively nonexpansive mapping is a relatively weak nonexpansive mapping (see [14]). In fact, for any mapping $T: C \rightarrow C$, we have $F(T) \subset$ $\tilde{F}(T) \subset \hat{F}(T)$. Therefore, if $T$ is a relatively nonexpansive mapping, then $F(T)=\tilde{F}(T)=$ $\hat{F}(T)$.

(2) The class of hemi-relatively nonexpansive mappings is more general than the class of relatively weak nonexpansive mappings.

The converse of Remark 1.2 is not true. In order to explain this better, we give the following example.

Example 1.1 ([24]) Let $E$ be any smooth Banach space, and let $x_{0} \neq 0$ be any element of $E$. We define a mapping $T: E \rightarrow E$ as follows:

$$
T x= \begin{cases}\left(\frac{1}{2}+\frac{1}{2^{n+1}}\right) x_{0} & \text { if } x=\left(\frac{1}{2}+\frac{1}{2^{n}}\right) x_{0} \\ -x & \text { if } x \neq\left(\frac{1}{2}+\frac{1}{2^{n}}\right) x_{0}\end{cases}
$$

for $n=1,2,3, \ldots$. Then $T$ is a hemi-relatively nonexpansive mapping but not a relatively nonexpansive mapping. 
Remark 1.3 There are other examples of hemi-relatively nonexpansive mappings such as the generalized projections (or projections) from a smooth, strictly convex and reflexive Banach space, and others; see [25].

A mapping $T: C \rightarrow C$ is said to be $c l o s e d$, if for any sequence $\left\{x_{n}\right\} \subset C$ with $x_{n} \rightarrow x$ and $T x_{n} \rightarrow y$, then $T x=y$.

In 2009, Kimura and Takahashi [26] proposed the following hybrid iteration method with a generalized projection for a family of relatively nonexpansive mappings $\left\{T_{\lambda}\right\}$ in a Banach space $E$ :

$$
\left\{\begin{array}{l}
x_{1}=x \in C, \quad \text { chosen arbitrarily, } \\
C_{1}=C, \\
y_{n}(\lambda)=J^{-1}\left(\alpha_{n} J x_{n}+\left(1-\alpha_{n}\right) J T_{\lambda} x_{n}\right) \quad \text { for all } \lambda \in \Lambda, \\
C_{n+1}=\left\{z \in C_{n}: \sup _{\lambda \in \Lambda} \phi\left(z, y_{n}(\lambda)\right) \leq \phi\left(z, x_{n}\right)\right\}, \\
x_{n+1}=\prod_{C_{n+1}} x .
\end{array}\right.
$$

They proved that $\left\{x_{n}\right\}$ converges strongly to $\Pi_{\mathcal{F}} \mathcal{X} \in C$, where $\mathcal{F}=\bigcap_{\lambda \in \Lambda} F\left(T_{\lambda}\right)$ is the set of common fixed points of $T_{\lambda}$, and $\Pi_{K}$ is the generalized projection of $E$ onto a nonempty closed convex subset $K$ of $E$.

Recently, Zegeye and Shahzad [14] introduced the following iterative scheme for finding a common element of the solution set of a variational inequality problem and a fixed point of a relatively weak nonexpansive mapping with $\gamma$-inverse strongly monotone mapping satisfying $\|A x\| \leq\|A x-A p\|$ for all $x \in C$ and $p \in V I(C, A)$ (see, e.g., [27]):

$$
\left\{\begin{array}{l}
x_{0} \in C, \quad \text { chosen arbitrarily, } \\
y_{n}=\Pi_{C}\left(J^{-1}\left(J x_{n}-\alpha_{n} A x_{n}\right)\right), \\
z_{n}=T y_{n}, \\
H_{0}=\left\{v \in C: \phi\left(v, z_{0}\right) \leq \phi\left(v, y_{0}\right) \leq \phi\left(v, x_{0}\right)\right\}, \\
W_{0}=C, \\
H_{n}=\left\{v \in H_{n-1} \cap W_{n-1}: \phi\left(v, z_{n}\right) \leq \phi\left(v, y_{n}\right) \leq \phi\left(v, x_{n}\right)\right\} \\
W_{n}=\left\{v \in H_{n-1} \cap W_{n-1}:\left\langle x_{n}-v, J x_{0}-J x_{n}\right\rangle \geq 0\right\}, \\
x_{n+1}=\Pi_{H_{n} \cap W_{n}} x_{0}, \quad n \geq 1 .
\end{array}\right.
$$

On the other hand, Qin et al. [25] proposed the following hybrid iterative scheme:

$$
\left\{\begin{array}{l}
x_{0} \in E, \quad \text { chosen arbitrarily, } \\
C_{1}=C, \\
x_{1}=\Pi_{C_{1} x_{0}}, \\
y_{n}=J^{-1}\left(\alpha_{n} J x_{n}+\left(1-\alpha_{n}\right) J T x_{n}\right), \\
u_{n} \in C \text { such that } f\left(u_{n}, y\right)+\frac{1}{r_{n}}\left\langle y-u_{n}, J u_{n}-J y_{n}\right\rangle \geq 0, \quad \forall y \in C, \\
C_{n+1}=\left\{z \in C_{n}: \phi\left(z, u_{n}\right) \leq \phi\left(z, x_{n}\right)\right\}, \\
x_{n+1}=\Pi_{C_{n+1}} x_{0},
\end{array}\right.
$$


where $T: C \rightarrow C$ is a closed hemi-relatively nonexpansive mapping. Under suitable conditions, they proved that the sequence $\left\{x_{n}\right\}$ converges strongly to $\prod_{F(T) \cap E P(f)} x_{0}$, where $E P(f)$ is the solution of an equilibrium problem for a bifunction $f: C \times C \rightarrow \mathbb{R}$.

In this paper, we introduce a new hybrid projection iterative scheme that converges strongly to a common element of the solution set of a generalized mixed equilibrium problem, the solution set of a variational inequality problem, and the set of common fixed points for a family of hemi-relatively nonexpansive mappings in a Banach space.

\section{Preliminaries}

Let $E$ be a normed linear space with $\operatorname{dim} E \geq 2$. The modulus of smoothness of $E$ is the function $\rho_{E}:[0, \infty) \rightarrow[0, \infty)$ defined by

$$
\rho_{E}(\tau):=\sup \left\{\frac{\|x+y\|+\|x-y\|}{2}-1:\|x\|=1,\|y\|=\tau\right\} .
$$

The space $E$ is said to be smooth if $\rho_{E}(\tau)>0, \forall \tau>0$ and $E$ is called uniformly smooth if and only if $\lim _{t \rightarrow 0} \frac{\rho_{E}(t)}{t}=0$. The modulus of convexity of $E$ is the function $\delta_{E}:(0,2] \rightarrow[0,1]$ defined by

$$
\delta_{E}(\varepsilon):=\inf \left\{1-\frac{\|x+y\|}{2}:\|x\|=\|y\|=1, \varepsilon=\|x-y\|\right\} .
$$

$E$ is called uniformly convex if and only if $\delta_{E}(\varepsilon)>0$ for every $\varepsilon \in(0,2]$. Let $p>1$. Then $E$ is said to be $p$-uniformly convex if there exists a constant $c>0$ such that $\delta(\varepsilon) \geq c \cdot \varepsilon^{p}$ for all $\varepsilon \in[0,2]$. Observe that every $p$-uniformly convex space is uniformly convex.

It is well known (see, for example, [28]) that

$$
L_{p}\left(l_{p}\right) \quad \text { or } \quad W_{m}^{p} \text { is } \begin{cases}p \text {-uniformly convex } & \text { if } p \geq 2 \\ 2 \text {-uniformly convex } & \text { if } 1<p \leq 2 .\end{cases}
$$

In the following, we shall need the following results.

Lemma 2.1 ([28]) Let E be a 2-uniformly convex and smooth Banach space. Then, for all $x, y \in E$, we have

$$
\|x-y\| \leq \frac{2}{c^{2}}\|x-J y\|
$$

where $J$ is the normalized duality mapping of $E$, and $\frac{1}{c}(0<c \leq 1)$ is the 2-uniformly convex constant of $E$.

Lemma $2.2([18,29])$ Let E be a real smooth, strictly convex and reflexive Banach space, and let $C$ be a nonempty closed convex subset of $E$. Then the following conclusions hold:

(i) $\phi\left(y, \Pi_{C} x\right)+\phi\left(\Pi_{C} x, x\right) \leq \phi(y, x), \forall x \in E, y \in C$.

(ii) Suppose $x \in E$ and $z \in C$. Then

$$
z=\Pi_{C} x \quad \Leftrightarrow \quad\langle z-y, J x-J z\rangle \geq 0, \quad \forall y \in C .
$$


Lemma 2.3 ([30]) Let E be a strictly convex and smooth Banach space, $C$ be a nonempty closed and convex subset of $E$ and $T: C \rightarrow C$ be a hemi-relatively nonexpansive mapping. Then $F(T)$ is a closed convex subset of $C$.

Lemma 2.4 ([29]) Let E be a real smooth and uniformly convex Banach space, and let $\left\{x_{n}\right\},\left\{y_{n}\right\}$ be two sequences of $E$. If $\lim _{n \rightarrow \infty} \phi\left(x_{n}, y_{n}\right)=0$ and either $\left\{x_{n}\right\}$ or $\left\{y_{n}\right\}$ is bounded, then $\lim _{n \rightarrow \infty}\left\|x_{n}-y_{n}\right\|=0$.

Lemma 2.5 ([31]) Let $E$ be a real smooth Banach space and $A: E \rightarrow E^{*}$ be a maximal monotone mapping. Then $A^{-1}(0)$ is a closed and convex subset of $E$.

We denote the normal cone for $C$ at a point $v \in C$ by $N_{C}(v)$, that is,

$$
N_{C}(v):=\left\{x^{*} \in E^{*}:\left\langle v-y, x^{*}\right\rangle \geq 0, \forall y \in C\right\} .
$$

Lemma 2.6 ([32]) Let $C$ be a nonempty closed convex subset of a Banach space E, and let $A$ be a monotone and hemicontinuous mapping of $C$ into $E^{*}$ with $C=D(A)$. Let $T \subset E \times E^{*}$ be a mapping defined as follows:

$$
T v:= \begin{cases}A v+N_{C}(v), & v \in C, \\ \emptyset, & v \notin C .\end{cases}
$$

Then $T$ is maximal monotone and $T^{-1}(0)=V I(C, A)$.

Remark 2.1 It is well known that the monotone and hemicontinuous mapping $A$ with $D(A)=E$ is maximal (see, e.g., [1]). Note that Lemma 2.6 is for the monotone and hemicontinuous mapping.

Remark 2.2 Let $C$ be a nonempty closed convex subset of a Banach space $E$, and let $A$ be a monotone and hemicontinuous mapping from $C$ into $E^{*}$ with $C=D(A)$. Then

$$
V I(C, A)=\{u \in C:\langle v-u, A v\rangle \geq 0 \text { for all } v \in C\} .
$$

It is obvious that the set $V I(C, A)$ is a closed convex subset of $C$ and the set $A^{-1} 0=V I(E, A)$ is a closed convex subset of $E$ (see [27]).

We make use of the function $V: E \times E^{*} \rightarrow \mathbb{R}$ defined by

$$
V\left(x, x^{*}\right)=\|x\|^{2}-2\left\langle x, x^{*}\right\rangle+\left\|x^{*}\right\|^{2}
$$

for all $x \in E$ and $x^{*} \in E^{*}$, which was studied by Alber [18]. That is,

$$
V\left(x, x^{*}\right)=\phi\left(x, J^{-1} x^{*}\right)
$$

for all $x \in E$ and $x^{*} \in E^{*}$. We know the following lemma. 
Lemma 2.7 ([18]) Let E be a reflexive, strictly convex and smooth Banach space with $E^{*}$ as its dual. Then

$$
V\left(x, x^{*}\right)+2\left\langle J^{-1} x^{*}-x, y^{*}\right) \leq V\left(x, x^{*}+y^{*}\right)
$$

for all $x \in E$ and $x^{*}, y^{*} \in E^{*}$.

Lemma 2.8 ([2]) Let C be a closed subset of a smooth, strictly convex and reflexive Banach space E. Let $B: C \rightarrow E^{*}$ be a continuous and monotone mapping, $\psi: C \rightarrow \mathbb{R}$ be a lower semicontinuous and convex function, and $\theta$ be a bifunction from $C \times C$ to $\mathbb{R}$ satisfying (E1)-(E4). Then, for $r>0$ and $x \in E$, there exists $u \in C$ such that

$$
\theta(u, y)+\langle B u, y-u\rangle+\psi(y)-\psi(u)+\frac{1}{r}\langle y-u, J u-J x\rangle \geq 0, \quad \forall y \in C .
$$

Define a mapping $T_{r}: E \rightarrow C$ by

$$
\begin{aligned}
T_{r}(x)= & \{u \in C: \theta(u, y)+\langle B u, y-u\rangle+\psi(y)-\psi(u) \\
& \left.+\frac{1}{r}\langle y-u, J u-J x\rangle \geq 0, \forall y \in C\right\}
\end{aligned}
$$

for all $x \in E$. Then the following conclusions hold:

(i) $T_{r}$ is single-valued;

(ii) $T_{r}$ is a firmly nonexpansive type mapping [33], i.e., for all $x, y \in E$,

$$
\left\langle T_{r} x-T_{r} y, J T_{r} x-J T_{r} y\right\rangle \leq\left\langle T_{r} x-T_{r} y, J x-J y\right\rangle
$$

(iii) $F\left(T_{r}\right)=G M E P=\hat{F}\left(T_{r}\right)$;

(iv) $G M E P$ is a closed and convex subset of $C$;

(v) $\phi\left(p, T_{r} z\right)+\phi\left(T_{r} z, z\right) \leq \phi(p, z), \forall p \in F\left(T_{r}\right), x \in E$.

Remark 2.3 ([2]) The mapping $T_{r}: E \rightarrow C$ defined by (2.1) is a relatively nonexpansive mapping. Thus, it is a hemi-relatively nonexpansive mapping.

\section{An iterative scheme for a family of hemi-relatively nonexpansive mappings}

In this section, we introduce a new hybrid iterative scheme for a common element of the solution set of problem (1.1), the solution set of problem (1.3) for an inverse strongly monotone mapping and the set of common fixed points of a family of hemi-relatively nonexpansive mappings.

Theorem 3.1 Let E be a real uniformly smooth and 2-uniformly convex Banach space and $C$ be a nonempty, closed and convex subset of $E$. Let $A: C \rightarrow E^{*}$ be a $\gamma$-inverse strongly monotone mapping and $B: C \rightarrow E^{*}$ be a continuous and monotone mapping. Let $\psi: C \rightarrow$ $\mathbb{R}$ be a lower semicontinuous and convex function and $\theta$ be a bifunction from $C \times C$ to $\mathbb{R}$ satisfying (E1)-(E4). Let $\left\{T_{\lambda}: \lambda \in \Lambda\right\}$ be a family of closed hemi-relatively nonexpansive mappings of $C$ into itself having

$$
\Omega=\mathcal{F} \cap V I(C, A) \cap G M E P \neq \emptyset,
$$


where $\mathcal{F}=\bigcap_{\lambda \in \Lambda} F\left(T_{\lambda}\right)$ is the set of common fixed points of $\left\{T_{\lambda}\right\}$. Assume that $\|A x\| \leq \| A x-$ Ap $\|$ for all $x \in C$ and $p \in V I(C, A)$. Suppose that $0<a<\mu_{n}<b=\frac{c^{2} \gamma}{2}$, where $c$ is the constant in Lemma 2.1. Let $\left\{r_{n}\right\} \subset\left[c^{*},+\infty\right)$ for some $c^{*}>0$. Let $\left\{x_{n}\right\}$ be the sequence generated by

$$
\left\{\begin{aligned}
x_{0} \in C, \quad \text { chosen arbitrarily, } & \\
y_{n}= & \Pi_{C} J^{-1}\left(J x_{n}-\mu_{n} A x_{n}\right), \\
z_{n, \lambda}= & J^{-1}\left(\alpha_{n} J x_{n}+\left(1-\alpha_{n}\right) J T_{\lambda} y_{n}\right), \\
u_{n, \lambda}= & T_{r_{n}, \lambda} z_{n, \lambda}, \\
H_{0, \lambda}= & \left\{v \in C: \sup _{\lambda \in \Lambda} \phi\left(v, u_{0, \lambda}\right)\right. \\
& \left.\leq \alpha_{0} \phi\left(v, x_{0}\right)+\left(1-\alpha_{0}\right) \phi\left(v, y_{0}\right) \leq \phi\left(v, x_{0}\right)\right\}, \\
H_{n, \lambda}= & \left\{v \in H_{n-1, \lambda} \cap W_{n-1, \lambda}: \sup _{\lambda \in \Lambda} \phi\left(v, u_{n, \lambda}\right)\right. \\
& \left.\leq \alpha_{n} \phi\left(v, x_{n}\right)+\left(1-\alpha_{n}\right) \phi\left(v, y_{n}\right) \leq \phi\left(v, x_{n}\right)\right\}, \\
W_{0, \lambda}= & C, \\
W_{n, \lambda}= & \left\{v \in H_{n-1, \lambda} \cap W_{n-1, \lambda}:\left\langle x_{n}-v, J x_{0}-J x_{n}\right\rangle \geq 0\right\}, \\
x_{n+1}= & \Pi_{H_{n, \lambda} \cap W_{n, \lambda} x_{0}, \quad n \geq 0, \lambda \in \Lambda,}
\end{aligned}\right.
$$

where $J$ is the normalized duality mapping, and $\left\{\alpha_{n}\right\}$ is a sequence in $[0,1]$ satisfying $\liminf _{n \rightarrow \infty}\left(1-\alpha_{n}\right)>0$. Then $\left\{x_{n}\right\}$ converges to $\Pi_{\Omega} x_{0}$, where $\Pi_{\Omega}$ is the generalized projection of $E$ onto $\Omega$.

Proof Step 1. We prove that $H_{n, \lambda}$ and $W_{n, \lambda}$ both are closed and convex subsets of $C$ and $\Omega \subset H_{n, \lambda} \cap W_{n, \lambda}$ with $n \geq 0, \lambda \in \Lambda$. In fact, it is obvious that $W_{n, \lambda}$ is closed and convex, and $H_{n, \lambda}$ is closed for each $n \geq 0, \lambda \in \Lambda$. Since

$$
\begin{aligned}
& \phi\left(v, u_{n, \lambda}\right) \leq \alpha_{n} \phi\left(v, x_{n}\right)+\left(1-\alpha_{n}\right) \phi\left(v, y_{n}\right) \\
& \quad \Leftrightarrow \quad 2\left\langle v, \alpha_{n} J x_{n}+\left(1-\alpha_{n}\right) J y_{n}-J u_{n, \lambda}\right\rangle \leq\left(1-\alpha_{n}\right)\left\|y_{n}\right\|^{2}-\left\|u_{n, \lambda}\right\|^{2}+\alpha_{n}\left\|x_{n}\right\|^{2}
\end{aligned}
$$

and

$$
\begin{aligned}
& \alpha_{n} \phi\left(v, x_{n}\right)+\left(1-\alpha_{n}\right) \phi\left(v, y_{n}\right) \leq \phi\left(v, x_{n}\right) \\
& \Leftrightarrow \quad \phi\left(v, y_{n}\right) \leq \phi\left(v, x_{n}\right),
\end{aligned}
$$

$H_{n, \lambda}$ is convex for each $n \geq 0, \lambda \in \Lambda$. Hence $H_{n, \lambda} \cap W_{n, \lambda}$ is closed and convex for all $n \geq 0$, $\lambda \in \Lambda$.

Step 2. For any given $p \in \Omega$, from (v) of Lemma 2.8 and that $T_{\lambda}$ is hemi-relatively nonexpansive, we have

$$
\begin{aligned}
\phi\left(p, u_{0, \lambda}\right)= & \phi\left(p, T_{r_{0}, \lambda} z_{0, \lambda}\right) \\
\leq & \phi\left(p, z_{0, \lambda}\right)=\phi\left(p, J^{-1}\left(\alpha_{0} J x_{0}+\left(1-\alpha_{0}\right) J T_{\lambda} y_{0}\right)\right) \\
= & \|p\|^{2}-2\left\langle p, \alpha_{0} J x_{0}+\left(1-\alpha_{0}\right) J T_{\lambda} y_{0}\right\rangle \\
& +\left\|\alpha_{0} J x_{0}+\left(1-\alpha_{0}\right) J T_{\lambda} y_{0}\right\|^{2} \\
\leq & \|p\|^{2}-2 \alpha_{0}\left\langle p, J x_{0}\right\rangle-2\left(1-\alpha_{0}\right)\left\langle p, J T_{\lambda} y_{0}\right\rangle
\end{aligned}
$$




$$
\begin{aligned}
& +\alpha_{0}\left\|x_{0}\right\|^{2}+\left(1-\alpha_{0}\right)\left\|T_{\lambda} y_{0}\right\|^{2} \\
= & \alpha_{0} \phi\left(p, x_{0}\right)+\left(1-\alpha_{0}\right) \phi\left(p, T_{\lambda} y_{0}\right) \\
\leq & \alpha_{0} \phi\left(p, x_{0}\right)+\left(1-\alpha_{0}\right) \phi\left(p, y_{0}\right)
\end{aligned}
$$

for each $\lambda \in \Lambda$. From Lemma 2.1, Lemma 2.7, and the assumption of $A$, we obtain

$$
\begin{aligned}
\phi\left(p, y_{0}\right)= & \phi\left(p, \Pi_{C} J^{-1}\left(J x_{0}-\mu_{0} A x_{0}\right)\right) \\
\leq & \phi\left(p, J^{-1}\left(J x_{0}-\mu_{0} A x_{0}\right)\right) \\
= & V\left(p, J x_{0}-\mu_{0} A x_{0}\right) \\
\leq & V\left(p, J x_{0}-\mu_{0} A x_{0}+\mu_{0} A x_{0}\right) \\
& -2\left\langle J^{-1}\left(J x_{0}-\mu_{0} A x_{0}\right)-p, \mu_{0} A x_{0}\right\rangle \\
= & V\left(p, J x_{0}\right)-2 \mu_{0}\left\langle J^{-1}\left(J x_{0}-\mu_{0} A x_{0}\right)-J^{-1}\left(J x_{0}\right), A x_{0}\right\rangle \\
& -2 \mu_{0}\left\langle x_{0}-p, A x_{0}\right\rangle \\
\leq & V\left(p, J x_{0}\right)-2 \mu_{0}\left\langle J^{-1}\left(J x_{0}-\mu_{0} A x_{0}\right)-J^{-1}\left(J x_{0}\right), A x_{0}\right\rangle \\
& -2 \mu_{0}\left\langle x_{0}-p, A x_{0}-A p\right\rangle-2 \mu_{0}\left\langle x_{0}-p, A p\right\rangle \\
\leq & \phi\left(p, x_{0}\right)+\frac{4 \mu_{0}^{2}}{c^{2}}\left\|A x_{0}\right\|^{2}-2 \mu_{0} \gamma\left\|A x_{0}-A p\right\|^{2} \\
\leq & \phi\left(p, x_{0}\right)+2 \mu_{0}\left(\frac{2 \mu_{0}}{c^{2}}-\gamma\right)\left\|A x_{0}-A p\right\|^{2} \\
\leq & \phi\left(p, x_{0}\right) .
\end{aligned}
$$

From (3.2) and (3.3),

$$
\phi\left(p, u_{0, \lambda}\right) \leq \alpha_{0} \phi\left(p, x_{0}\right)+\left(1-\alpha_{0}\right) \phi\left(p, y_{0}\right) \leq \phi\left(p, x_{0}\right)
$$

for each $\lambda \in \Lambda$. Thus

$$
\sup _{\lambda \in \Lambda} \phi\left(p, u_{0, \lambda}\right) \leq \alpha_{0} \phi\left(p, x_{0}\right)+\left(1-\alpha_{0}\right) \phi\left(p, y_{0}\right) \leq \phi\left(p, x_{0}\right) .
$$

Therefore, $p \in H_{0, \lambda}$ and $p \in H_{0, \lambda} \cap W_{0, \lambda}$. Suppose that $\Omega \subset H_{n-1, \lambda} \cap W_{n-1, \lambda}$. Then, the methods in (3.2) and (3.3) imply that

$$
\phi\left(p, u_{n, \lambda}\right) \leq \alpha_{n} \phi\left(p, x_{n}\right)+\left(1-\alpha_{n}\right) \phi\left(p, y_{n}\right) \leq \phi\left(p, x_{n}\right), \quad \forall \lambda \in \Lambda,
$$

which implies that $p \in H_{n, \lambda}$. Since $x_{n}=\Pi_{H_{n-1, \lambda} \cap W_{n-1, \lambda}} x_{0}$, it follows from Lemma 2.2 that

$$
\left\langle x_{n}-z, J x_{0}-J x_{n}\right\rangle \geq 0, \quad \forall z \in H_{n-1, \lambda} \cap W_{n-1, \lambda} .
$$

It implies that $\left\langle x_{n}-p, J x_{0}-J x_{n}\right\rangle \geq 0$. Hence $p \in W_{n, \lambda}$. Therefore, $\Omega \subset H_{n, \lambda} \cap W_{n, \lambda}$. Then, by induction on $n, \Omega \subset H_{n, \lambda} \cap W_{n, \lambda}$ for all $n \geq 0, \lambda \in \Lambda$. From Lemma 2.5 and Lemma 2.6, we know that $\operatorname{VI}(C, A)$ is closed and convex set. Therefore, $\Omega$ is closed and convex. The sequence $\left\{x_{n}\right\}$ generated by (3.1) is well defined. 
Step 3. We prove that $\left\{x_{n}\right\}$ is a Cauchy sequence. Let $p \in \Omega$. From the definition of $H_{n, \lambda}$, $W_{n, \lambda}$ and Lemma 2.2, we have $x_{n}=\Pi_{H_{n-1, \lambda} \cap W_{n-1, \lambda}} x_{0}$ and

$$
\phi\left(p, x_{n}\right)+\phi\left(x_{n}, x_{0}\right) \leq \phi\left(p, x_{0}\right) .
$$

Thus $\left\{x_{n}\right\}$ is bounded. Moreover, since

$$
x_{n}=\Pi_{H_{n-1, \lambda} \cap W_{n-1, \lambda}} x_{0}, \quad x_{n+1}=\Pi_{H_{n, \lambda} \cap W_{n, \lambda}} x_{0} \in H_{n, \lambda} \cap W_{n, \lambda},
$$

we have

$$
\phi\left(x_{n+1}, x_{n}\right)+\phi\left(x_{n}, x_{0}\right) \leq \phi\left(x_{n+1}, x_{0}\right)
$$

which implies that $\left\{\phi\left(x_{n}, x_{0}\right)\right\}$ is nondecreasing. It follows that the limit of $\left\{\phi\left(x_{n}, x_{0}\right)\right\}$ exists. By the construction $H_{n, \lambda} \cap W_{n, \lambda}$, one has that

$$
\begin{aligned}
& H_{m, \lambda} \cap W_{m, \lambda} \subset H_{m-1, \lambda} \cap W_{m-1, \lambda}, \\
& x_{m}=\Pi_{H_{m, \lambda} \cap W_{m, \lambda}} x_{0} \in H_{n, \lambda} \cap W_{n, \lambda}
\end{aligned}
$$

for any positive integer $m \geq n$. From Lemma 2.2, we have

$$
\begin{aligned}
\phi\left(x_{m}, x_{n}\right) & =\phi\left(x_{m}, \Pi_{H_{n-1, \lambda} \cap W_{n-1, \lambda}} x_{0}\right) \\
& \leq \phi\left(x_{m}, x_{0}\right)-\phi\left(\Pi_{H_{n, \lambda} \cap W_{n, \lambda}} x_{0}, x_{0}\right) \\
& =\phi\left(x_{m}, x_{0}\right)-\phi\left(x_{n}, x_{0}\right) .
\end{aligned}
$$

Letting $m, n \rightarrow \infty$ in (3.5), we have

$$
\phi\left(x_{m}, x_{n}\right) \rightarrow 0
$$

Thus, Lemma 2.4 implies that

$$
\lim _{m, n \rightarrow \infty}\left\|x_{m}-x_{n}\right\|=0
$$

This implies that $\left\{x_{n}\right\}$ is a Cauchy sequence.

Step 4. Now, we prove that $\lim _{n \rightarrow \infty}\left\|y_{n}-T_{\lambda} y_{n}\right\|=0$ for each $\lambda \in \Lambda$ and $q \in \mathcal{F}=$ $\bigcap_{\lambda \in \Lambda} F\left(T_{\lambda}\right)$. Since $x_{n+1} \in H_{n, \lambda}$, we obtain

$$
\phi\left(x_{n+1}, u_{n, \lambda}\right) \leq \phi\left(x_{n+1}, x_{n}\right)
$$

for all $\lambda \in \Lambda$ and

$$
\phi\left(x_{n+1}, y_{n}\right) \leq \phi\left(x_{n+1}, x_{n}\right) .
$$

By (3.5) and Lemma 2.4, we have

$$
\lim _{n \rightarrow \infty}\left\|x_{n+1}-u_{n, \lambda}\right\|=\lim _{n \rightarrow \infty}\left\|x_{n+1}-y_{n}\right\|=0, \quad \forall \lambda \in \Lambda .
$$


Hence,

$$
\lim _{n \rightarrow \infty}\left\|x_{n}-u_{n, \lambda}\right\|=\lim _{n \rightarrow \infty}\left\|x_{n}-y_{n}\right\|=\lim _{n \rightarrow \infty}\left\|u_{n, \lambda}-y_{n}\right\|=0
$$

for all $\lambda \in \Lambda$. By the methods in (3.2) and (3.4), we have

$$
\phi\left(p, u_{n, \lambda}\right) \leq \phi\left(p, z_{n, \lambda}\right) \leq \phi\left(p, x_{n}\right)
$$

for all $n \geq 0, \lambda \in \Lambda$. Since $J$ is uniformly continuous on the bounded sets, it follows from Lemma $2.8(\mathrm{v}),(3.2)$ and (3.7) that for any given $p \in \Omega$,

$$
\begin{aligned}
\phi\left(u_{n, \lambda}, z_{n, \lambda}\right) & =\phi\left(T_{r_{n}, \lambda} z_{n, \lambda}, z_{n, \lambda}\right) \\
& \leq \phi\left(p, z_{n, \lambda}\right)-\phi\left(p, T_{r_{n}, \lambda} z_{n, \lambda}\right) \\
& \leq \phi\left(p, x_{n}\right)-\phi\left(p, u_{n, \lambda}\right) \\
& =\left\|x_{n}\right\|^{2}-\left\|u_{n, \lambda}\right\|^{2}-2\left\langle p, J x_{n}-J u_{n, \lambda}\right\rangle \\
& \leq\left(\left\|x_{n}\right\|-\left\|u_{n, \lambda}\right\|\right)\left(\left\|x_{n}\right\|+\left\|u_{n, \lambda}\right\|\right)+2\|p\|\left\|J x_{n}-J u_{n, \lambda}\right\| \\
& \rightarrow 0, \quad \text { as } n \rightarrow \infty
\end{aligned}
$$

for all $\lambda \in \Lambda$. From Lemma 2.4,

$$
\lim _{n \rightarrow \infty}\left\|u_{n, \lambda}-z_{n, \lambda}\right\|=0
$$

Thus $\lim _{n \rightarrow \infty}\left\|z_{n, \lambda}-x_{n}\right\|=0$ for each $\lambda \in \Lambda$. Since $J$ is uniformly continuous on bounded sets, we obtain

$$
\begin{aligned}
\left(1-\alpha_{n}\right)\left\|J x_{n}-J T_{\lambda} y_{n}\right\| & =\left\|\alpha_{n} J x_{n}+\left(1-\alpha_{n}\right) J T_{\lambda} y_{n}-J x_{n}\right\| \\
& =\left\|J z_{n, \lambda}-J x_{n}\right\| \\
& \rightarrow 0
\end{aligned}
$$

as $n \rightarrow \infty$. Since $\liminf _{n \rightarrow \infty}\left(1-\alpha_{n}\right)>0$ and $J^{-1}$ is uniformly continuous on bounded sets, we obtain

$$
\lim _{n \rightarrow \infty}\left\|x_{n}-T_{\lambda} y_{n}\right\|=0
$$

for all $\lambda \in \Lambda$. It follows from (3.6) and (3.9) that

$$
\left\|y_{n}-T_{\lambda} y_{n}\right\| \leq\left\|y_{n}-x_{n}\right\|+\left\|x_{n}-T_{\lambda} y_{n}\right\| \rightarrow 0, \quad \text { as } n \rightarrow \infty
$$

for all $\lambda \in \Lambda$. Since $\left\{x_{n}\right\}$ is a Cauchy sequence, there exists a point $q \in C$ such that $\lim _{n \rightarrow \infty} x_{n}=q$. It follows from (3.6) that $y_{n} \rightarrow q$. Since $T_{\lambda}$ is closed, from (3.10) we get $q \in \mathcal{F}=\bigcap_{\lambda \in \Lambda} F\left(T_{\lambda}\right)$.

Step 5. Now, we show that $q \in V I(C, A) \cap G M E P$. Let $S \subset E \times E^{*}$ be a mapping as follows:

$$
S v= \begin{cases}A v+N_{C}(v), & v \in C, \\ \emptyset, & v \notin C .\end{cases}
$$


By Lemma 2.6, $S$ is maximal monotone, and $S^{-1}(0)=V I(C, A)$. Let $(v, w) \in G(S)$ (graph of $S)$. Since $w \in S v=A v+N_{C}(v)$, we have $w-A v \in N_{C}(v)$. Moreover, $y_{n} \in C$ implies that

$$
\left\langle v-y_{n}, w-A v\right\rangle \geq 0 .
$$

On the other hand, from $y_{n}=\Pi_{C} J^{-1}\left(J x_{n}-\mu_{n} A x_{n}\right)$ and Lemma 2.2, we obtain that

$$
\left\langle v-y_{n}, J y_{n}-\left(J x_{n}-\mu_{n} A x_{n}\right)\right\rangle \geq 0 .
$$

Hence,

$$
\left\langle v-y_{n}, \frac{J x_{n}-J y_{n}}{\mu_{n}}-A x_{n}\right\rangle \leq 0 \text {. }
$$

From (3.11) and (3.12), we obtain

$$
\begin{aligned}
\left\langle v-y_{n}, w\right\rangle & \geq\left\langle v-y_{n}, A v\right\rangle \\
& \geq\left\langle v-y_{n}, A v\right\rangle+\left\langle v-y_{n}, \frac{J x_{n}-J y_{n}}{\mu_{n}}-A x_{n}\right\rangle \\
& =\left\langle v-y_{n}, A v-A x_{n}+\frac{J x_{n}-J y_{n}}{\mu_{n}}\right\rangle \\
& =\left\langle v-y_{n}, A v-A y_{n}\right\rangle+\left\langle v-y_{n}, A y_{n}-A x_{n}\right\rangle+\left\langle v-y_{n}, \frac{J x_{n}-J y_{n}}{\mu_{n}}\right\rangle \\
& \geq-\left\|v-y_{n}\right\|\left\|A y_{n}-A x_{n}\right\|-\left\|v-y_{n}\right\|\left\|\frac{J x_{n}-J y_{n}}{\mu_{n}}\right\| \\
& \geq-\frac{1}{\gamma}\left\|v-y_{n}\right\|\left\|y_{n}-x_{n}\right\|-\frac{1}{\mu_{n}}\left\|v-y_{n}\right\|\left\|J x_{n}-J y_{n}\right\| .
\end{aligned}
$$

Since $J$ is uniformly continuous on bounded sets, by (3.6) we have

$$
\lim _{n \rightarrow \infty}\left\langle v-y_{n}, w\right\rangle=\langle v-q, w\rangle \geq 0 .
$$

Thus, since $S$ is maximal monotone, we have $q \in S^{-1}(0)$ and $q \in V I(C, A)$. Next, we show that $q \in G M E P=F\left(T_{r, \lambda}\right)$. Let

$$
H_{\lambda}\left(u_{n, \lambda}, y\right)=\theta_{\lambda}\left(u_{n, \lambda}, y\right)+\left\langle B u_{n, \lambda}, y-u_{n, \lambda}\right\rangle+\psi(y)-\psi\left(u_{n, \lambda}\right), \quad \forall y \in C .
$$

From (3.6) and (3.8), we obtain $\lim _{n \rightarrow \infty} u_{n, \lambda}=q$ and $\lim _{n \rightarrow \infty} z_{n, \lambda}=q$ for all $\lambda \in \Lambda$. Since $J$ is uniformly continuous, from (3.8) we have $\lim _{n \rightarrow \infty}\left\|J u_{n, \lambda}-J z_{n, \lambda}\right\|=0$. Therefore, it follows from $r_{n} \in\left[c^{*}, \infty\right)$ for some $c^{*}>0$ that $\lim _{n \rightarrow \infty} \frac{\left\|u_{n, \lambda}-J z_{n, \lambda}\right\|}{r_{n}}=0$. Since $u_{n, \lambda}=T_{r_{n}, \lambda} z_{n, \lambda}$, we have

$$
H_{\lambda}\left(u_{n, \lambda}, y\right)+\frac{1}{r_{n}}\left\langle y-u_{n, \lambda}, J u_{n, \lambda}-J z_{n, \lambda}\right\rangle \geq 0, \quad \forall y \in C, \lambda \in \Lambda .
$$

Combining the above inequality and (E2), we get

$$
\begin{aligned}
\left\|y-u_{n, \lambda}\right\| \cdot \frac{\left\|J u_{n, \lambda}-J z_{n, \lambda}\right\|}{r_{n}} & \geq \frac{1}{r_{n}}\left\langle y-u_{n, \lambda}, J u_{n, \lambda}-J z_{n, \lambda}\right\rangle=-H_{\lambda}\left(u_{n, \lambda}, y\right) \\
& \geq H_{\lambda}\left(y, u_{n, \lambda}\right), \quad \forall y \in C, \lambda \in \Lambda .
\end{aligned}
$$


Taking the limit as $n \rightarrow \infty$ in the above inequality and by (E4), we have $H_{\lambda}(y, q) \leq 0$ for all $y \in C, \lambda \in \Lambda$. For any $t \in(0,1)$ and $y \in C$, define

$$
y_{t}=t y+(1-t) q \in C
$$

Then $H_{\lambda}\left(y_{t}, q\right) \leq 0$. From (E1) and (E4), we have

$$
\begin{aligned}
0 & =H_{\lambda}\left(y_{t}, y_{t}\right)=H_{\lambda}\left(y_{t}, t y+(1-t) q\right) \\
& \leq t H_{\lambda}\left(y_{t}, y\right)+(1-t) H_{\lambda}\left(y_{t}, q\right) \\
& \leq t H_{\lambda}\left(y_{t}, y\right)
\end{aligned}
$$

i.e., $H_{\lambda}\left(y_{t}, y\right) \geq 0$, for all $\lambda \in \Lambda$. Thus, from (E3) and let $t \downarrow 0$, we have $H_{\lambda}(q, y) \geq 0$ for all $y \in C, \lambda \in \Lambda$. This implies that $q \in G M E P$. Therefore $q \in \Omega$.

Step 6. Finally, we prove that $q=\Pi_{\Omega} x_{0}$. Since $x_{n+1}=\Pi_{H_{n, \lambda} \cap W_{n, \lambda}} x_{0}$ and by Lemma 2.2, we have

$$
\left\langle x_{n+1}-z, J x_{0}-J x_{n+1}\right\rangle \geq 0, \quad \forall z \in H_{n, \lambda} \cap W_{n, \lambda} .
$$

Taking the limit in (3.13) and from $\Omega \subset H_{n, \lambda} \cap W_{n, \lambda}$ for all $n \geq 0, \lambda \in \Lambda$, we obtain

$$
\left\langle q-z, J x_{0}-J q\right\rangle \geq 0, \quad \forall z \in \Omega
$$

Therefore, from Lemma 2.2, we have $q=\Pi_{\Omega} x_{0}$.

Remark 3.1 An iterative scheme for finding a solution of the variational inequality problem for a mapping $A$ that satisfies the following conditions in a 2-uniformly and uniformly smooth Banach space $E$ :

(1) $A$ is inverse strongly monotone,

(2) $V I(C, A) \neq \emptyset$,

(3) $\|A x\| \leq\|A x-A u\|$ for all $x \in C$ and $u \in V I(C, A)$.

If condition (3) holds, then we can prove a convergence theorem for variational inequality problems. To consider the general variational inequality problem for inverse strongly monotone mappings, we have to assume condition (3) (see [27]).

For a practical case, we may apply this theorem to a finite number of mappings $\left\{T_{1}, T_{2}, \ldots, T_{m}\right\}$ as follows.

Corollary 3.1 Let E be a real uniformly smooth and 2-uniformly convex Banach space and $C$ be a nonempty, closed and convex subset of $E$. Let $A: C \rightarrow E^{*}$ be a $\gamma$-inverse strongly monotone mapping and $B: C \rightarrow E^{*}$ be a continuous and monotone mapping. Let $\psi: C \rightarrow \mathbb{R}$ be a lower semicontinuous and convex function and $\theta$ be a bifunction from $C \times C$ to $\mathbb{R}$ satisfying (E1)-(E4). Let $\left\{T_{1}, T_{2}, \ldots, T_{m}\right\}$ be a finite family of closed hemi-relatively nonexpansive mappings of $C$ into itself having

$$
\Omega=\mathcal{F} \cap V I(C, A) \cap G M E P \neq \emptyset,
$$

where $\mathcal{F}=\bigcap_{k=1}^{m} F\left(T_{k}\right)$ is the set of common fixed points. Assume that $\|A x\| \leq\|A x-A p\|$ for all $x \in C$ and $p \in V I(C, A)$. Suppose that $0<a<\mu_{n}<b=\frac{c^{2} \gamma}{2}$, where $c$ is the constant in 
Lemma 2.1. Let $\left\{r_{n}\right\} \subset\left[c^{*},+\infty\right)$ for some $c^{*}>0$. Let $\left\{x_{n}\right\}$ be the sequence generated by

$$
\left\{\begin{aligned}
x_{0} \in C, \quad \text { chosen arbitrarily, } & \\
y_{n}= & \Pi_{C} J^{-1}\left(J x_{n}-\mu_{n} A x_{n}\right), \\
z_{n, k}= & J^{-1}\left(\alpha_{n} J x_{n}+\left(1-\alpha_{n}\right) J T_{k} y_{n}\right), \\
u_{n, k}= & T_{r_{n}, k} z_{n, k}, \\
H_{0, k}= & \left\{v \in C: \max _{k=1,2, \ldots, m} \phi\left(v, u_{0, k}\right)\right. \\
& \left.\leq \alpha_{0} \phi\left(v, x_{0}\right)+\left(1-\alpha_{0}\right) \phi\left(v, y_{0}\right) \leq \phi\left(v, x_{0}\right)\right\}, \\
H_{n, k}= & \left\{v \in H_{n-1, k} \cap W_{n-1, k}: \max _{k=1,2, \ldots, m} \phi\left(v, u_{n, k}\right)\right. \\
& \left.\leq \alpha_{n} \phi\left(v, x_{n}\right)+\left(1-\alpha_{n}\right) \phi\left(v, y_{n}\right) \leq \phi\left(v, x_{n}\right)\right\}, \\
W_{0, k}= & C, \\
W_{n, k}= & \left\{v \in W_{n-1, k} \cap H_{n-1, k}:\left\langle x_{n}-v, J x_{0}-J x_{n}\right\rangle \geq 0\right\}, \\
x_{n+1}= & \Pi_{H_{n, k} \cap W_{n, k} x_{0}, \quad n \geq 0, k=1,2, \ldots, m,}
\end{aligned}\right.
$$

where $J$ is the normalized duality mapping, and $\left\{\alpha_{n}\right\}$ is a sequence in $[0,1]$ satisfying $\liminf _{n \rightarrow \infty}\left(1-\alpha_{n}\right)>0$. Then $\left\{x_{n}\right\}$ converges to $\Pi_{\Omega} x_{0}$, where $\Pi_{\Omega}$ is the generalized projection of $E$ onto $\Omega$.

If $E=H$ is the Hilbert space, then $J=J^{-1}=I$ is the identity mapping on $H$. Then Theorem 3.1 reduces to the following corollary.

Corollary 3.2 Let $H$ be a real Hilbert space and $C$ be a nonempty, closed and convex subset of $H$. Let $A: C \rightarrow H$ be a continuous and monotone mapping. Let $\psi: C \rightarrow \mathbb{R}$ be a lower semicontinuous and convex function and $\theta$ be a bifunction from $C \times C$ to $\mathbb{R}$ satisfying (E1)(E4). Let $\left\{T_{\lambda}: C \rightarrow C: \lambda \in \Lambda\right\}$ be a family of closed hemi-relatively nonexpansive mappings with

$$
\Omega=\mathcal{F} \cap V I(C, A) \cap G M E P \not \emptyset
$$

where $\mathcal{F}=\bigcap_{\lambda \in \Lambda} F\left(T_{\lambda}\right)$ is the set of common fixed points of $\left\{T_{\lambda}\right\}$. Assume that $\|A x\| \leq \| A x-$ Ap $\|$ for all $x \in C$ and $p \in V I(C, A)$. Suppose that $0<a<\mu_{n}<b=\frac{c^{2} \gamma}{2}$, where $c$ is the constant in Lemma 2.1. Let $\left\{r_{n}\right\} \subset\left[c^{*},+\infty\right)$ for some $c^{*}>0$. Let $\left\{x_{n}\right\}$ be the sequence generated by

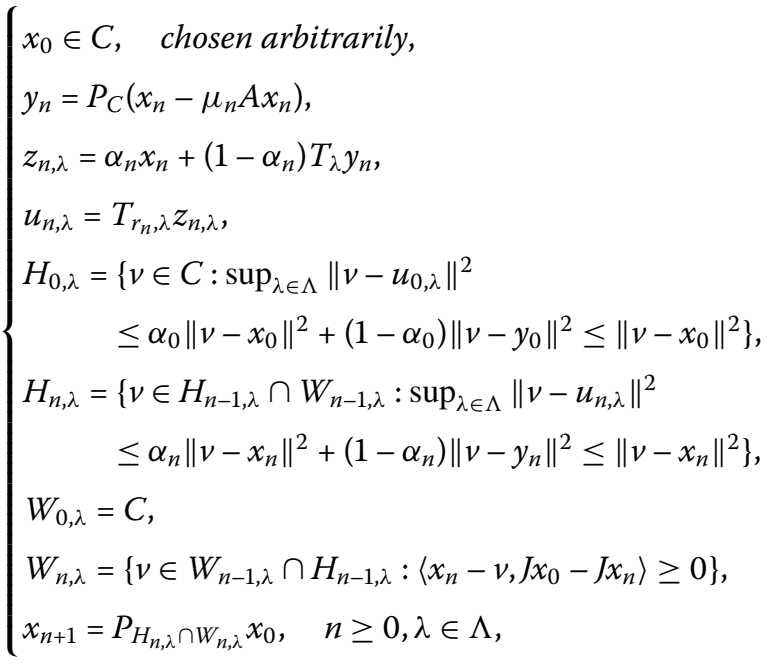


where $\left\{\alpha_{n}\right\}$ is a sequence in $[0,1]$ satisfying $\liminf _{n \rightarrow \infty}\left(1-\alpha_{n}\right)>0$. Then $\left\{x_{n}\right\}$ converges to $P_{\Omega} x_{0}$, where $P_{\Omega}$ is the metric projection of $H$ onto $\Omega$.

\section{Competing interests}

The authors declare that they have no competing interests.

\section{Authors' contributions}

The main idea of this paper was proposed by KSK. KSK prepared the manuscript initially, JKK and WHL confirmed all the steps of proofs in this research. All authors read and approved the final manuscript.

\section{Author details}

${ }_{1}^{1}$ Graduate School of Education, Mathematics Education, Kyungnam University, Changwon, Kyungnam 631-701, Republic of Korea. ${ }^{2}$ Department of Mathematics Education, Kyungnam University, Changwon, Kyungnam 631-701, Republic of Korea.

\section{Acknowledgements}

The authors would like to thank the referees for their valuable comments and suggestions which improved the presentation of this paper. This research was supported by the Basic Science Research Program through the National Research Foundation of Korea (NRF) funded by the Ministry of Education (2012R1A1A4A01010526)

Received: 12 July 2013 Accepted: 1 December 2013 Published: 02 Jan 2014

\section{References}

1. Cioranescu, I: Geometry of Banach Spaces, Duality Mappings and Nonlinear Problems, pp. 111-121. Kluwer Academic, Amsterdam (1990)

2. Zhang, SS: Generalized mixed equilibrium problem in Banach spaces. Appl. Math. Mech. 30(9), 1105-1112 (2009)

3. Ceng, LC, Yao, JC: A hybrid iterative scheme for mixed equilibrium problems and fixed point problems. J. Comput. Appl. Math. 214, 186-201 (2008)

4. Takahashi, S, Takahashi, W: Strong convergence theorem for a generalized equilibrium problem and a nonexpansive mapping in a Hilbert space. Nonlinear Anal. 69, 1025-1033 (2008)

5. Blum, E, Oettli, W: From optimization and variational inequalities to equilibrium problems. Math. Stud. 63, 123-145 (1994)

6. Chang, SS, Lee, HWJ, Chan, CK: A new hybrid method for solving a generalized equilibrium problem, solving a variational inequality problem and obtaining common fixed points in Banach spaces with applications. Nonlinear Anal. 73, 2260-2270 (2010)

7. Ibaraki, T, Kimura, Y, Takahashi, W: Convergence theorems for generalized projections and maximal monotone operators in Banach spaces. Abstr. Appl. Anal. 2003(10), 621-629 (2003)

8. Kim, JK, Kim, KS: New systems of generalized mixed variational inequalities with nonlinear mappings in Hilbert spaces. J. Comput. Anal. Appl. 12(3), 601-612 (2010)

9. Kim, JK, Kim, KS: On new systems of generalized nonlinear mixed quasivariational inequalities with two-variable operators. Taiwan. J. Math. 11(3), 867-881 (2007)

10. Kim, JK, Kim, KS: A new system of a generalized nonlinear mixed quasivariational inequalities and convergence in Hilbert spaces. J. Korean Math. Soc. 44(4), 823-834 (2007)

11. Kim, KS: Convergence to common solutions of various problems for nonexpansive mappings in Hilbert spaces. Fixed Point Theory Appl. 2012, 185 (2012)

12. Matsushita, S, Takahashi, W: A strong convergence theorem for relatively nonexpansive mappings in a Banach space. J. Approx. Theory 134, 257-266 (2005)

13. Takahashi, W, Zembayashi, K: Strong and weak convergence theorems for equilibrium problems and relatively nonexpansive mappings in Banach spaces. Nonlinear Anal. 70, 45-57 (2009)

14. Zegeye, H, Shahzad, N: Strong convergence theorems for monotone mappings and relatively weak nonexpansive mappings. Nonlinear Anal. 70, 2707-2716 (2009)

15. Kim, KS: Convergence of a hybrid algorithm for a reversible semigroup of nonlinear operators in Banach spaces. Nonlinear Anal. 73, 3413-3419 (2010)

16. Kim, KS: Nonlinear ergodic theorems of nonexpansive type mappings. J. Math. Anal. Appl. 358, 261-272 (2009)

17. Kim, KS: Approximation of common fixed points for an implicit iteration with errors of finite family of nonself nonexpansive mappings in Banach spaces. Dyn. Contin. Discrete Impuls. Syst., Ser. B, Appl. Algorithms 15(6), 807-815 (2008)

18. Alber, Yl: Metric and generalized projection operators in Banach spaces: properties and applications. In: Kartsatos, AG (ed.) Theory and Applications of Nonlinear Operators of Accretive and Monotone Type, pp. 15-50. Dekker, New York (1996)

19. Alber, Yl, Reich, S: An iterative method for solving a class of nonlinear operator equations in Banach spaces. Panam. Math. J. 4(2), 39-54 (1994)

20. Reich, S: A weak convergence theorem for the alternating method with Bregman distance. In: Kartsatos, AG (ed.) Theory and Applications of Nonlinear Operators of Accretive and Monotone Type, pp. 313-318. Dekker, New York (1996)

21. Butnariu, D, Reich, S, Zaslavski, AJ: Weak convergence of orbits of nonlinear operators in reflexive Banach spaces. Numer. Funct. Anal. Optim. 24, 489-508 (2003)

22. Butnariu, D, Reich, S, Zaslavski, AJ: Asymptotic behavior of relatively nonexpansive operators in reflexive Banach spaces. J. Appl. Anal. 7, 151-174 (2001) 
23. Censor, Y, Reich, S: Iterations of paracontractions and firmly nonexpansive operators with applications to feasibility and optimization. Optimization 37, 323-339 (1996)

24. Su, Y, Wang, Z, Xu, HK: Strong convergence theorems for a common fixed point of two hemi-relatively nonexpansive mappings. Nonlinear Anal. 71(11), 5616-5628 (2009)

25. Qin, X, Cho, YJ, Kang, SM: Convergence theorems of common elements for equilibrium problems and fixed point problems in Banach spaces. J. Comput. Appl. Math. 225(1), 20-30 (2009)

26. Kimura, Y, Takahashi, W: On a hybrid method for a family of relatively nonexpansive mappings in Banach space. J. Math. Anal. Appl. 357, 356-363 (2009)

27. liduka, $\mathrm{H}$, Takahashi, W: Strong convergence studied by a hybrid type method for monotone operators in a Banach space. Nonlinear Anal. 68, 3679-3688 (2008)

28. Xu, HK: Inequalities in Banach spaces with applications. Nonlinear Anal. 16, 1127-1138 (1991)

29. Kamimura, S, Takahashi, W: Strong convergence of a proximal-type algorithm in a Banach space. SIAM J. Optim. 13, 938-945 (2002)

30. Kim, JK: Strong convergence theorems by hybrid projection methods for equilibrium problems and fixed point problems of the asymptotically quasi- $\varphi$-nonexpansive mappings. Fixed Point Theory Appl. 2011, 10 (2011)

31. Pascali, D, Sburlan, S: Nonlinear Mappings of Monotone Type. Editura Academiae, Bucaresti (1978)

32. Rockfeller, RT: Monotone operators and the proximal point algorithm. SIAM J. Control Optim. 14, $877-898$ (1976)

33. Kohsaka, F, Takahashi, W: Existence and approximation of fixed points of firmly nonexpansive type mappings in Banach spaces. SIAM J. Optim. 19(2), 824-835 (2008)

10.1186/1029-242X-2014-2

Cite this article as: Kim et al.: Convergence theorems for common solutions of various problems with nonlinear mapping. Journal of Inequalities and Applications 2014, 2014:2

\section{Submit your manuscript to a SpringerOpen ${ }^{\circ}$ journal and benefit from:}

- Convenient online submission

- Rigorous peer review

- Immediate publication on acceptance

- Open access: articles freely available online

- High visibility within the field

- Retaining the copyright to your article 